\title{
Trastuzumab and fulvestrant combination therapy for women with advanced breast cancer positive for hormone receptor and human epidermal growth factor receptor 2: a retrospective single-center study
}

\author{
Yukinori Ozaki ${ }^{1}{ }^{2 *}$, Yosuke Aoyama ${ }^{3}$, Jun Masuda ${ }^{1}$, Lina Inagaki ${ }^{1}$, Saori Kawai ${ }^{1}$, Tomoko Shibayama ${ }^{1}$, \\ Tetsuyo Maeda', Mami Kurata', Kazuyo Yoshida', Sumito Saeki', Mari Hosonaga', Ippei Fukada', \\ Fumikata Hara' ${ }^{1}$ Takayuki Kobayashi ${ }^{1}$, Kokoro Kobayashi $^{1}$, Satoshi Miyake², Toshimi Takano', \\ Takayuki Ueno ${ }^{1}$ and Shinji Ohno
}

\begin{abstract}
Background: Trastuzumab and fulvestrant combination therapy is one of the treatment options for patients with hormone receptor- and human epidermal growth factor receptor 2 (HER2)-positive metastatic breast cancer; however, there are limited studies evaluating the efficacy of this combination therapy.
\end{abstract}

Methods: We retrospectively reviewed the data of women with hormone receptor- and HER2-positive metastatic breast cancer who received trastuzumab and fulvestrant combination therapy between August 1997 and August 2020 at the Cancer Institute Hospital. The primary endpoint of this study was progression-free survival, and the secondary endpoints were response rate, overall survival and safety.

Results: We reviewed the data of 1612 patients with recurrent or metastatic breast cancer, of which 118 patients were diagnosed with hormone receptor- and HER2-positive breast cancer. Of these, 28 patients who received trastuzumab and fulvestrant combination therapy were eligible for this study. The median treatment line for advanced breast cancer was 6 (range, 1-14), the median progression-free survival was 6.4 months ( $95 \%$ confidence interval [Cl], 3.46-8.17), and the median overall survival was 35.3 months ( $95 \% \mathrm{Cl}, 20.0-46.7)$. Of the 28 patients, partial response was observed in 1 (4\%), stable disease in 17 (61\%), and progressive disease in $10(36 \%)$ patients. The disease control rate was $64 \%$. Adverse events of grade $\geq 3$ were not observed.

Conclusions: Trastuzumab and fulvestrant combination therapy showed moderate clinical efficacy and no severe toxicity after standard anti-HER2 treatment, which is a reasonable treatment option for patients with hormone receptor- and HER2-positive metastatic breast cancer. These data contribute to understanding the efficacy of trastuzumab

\footnotetext{
*Correspondence: yukinori.ozaki@jfcr.or.jp

1 Breast Oncology Center, The Cancer Institute Hospital of Japanese

Foundation for Cancer Research, 3-8-31 Ariake, Koto-ku, Tokyo 135-8550,

Japan

Full list of author information is available at the end of the article
}

(c) The Author(s) 2022. Open Access This article is licensed under a Creative Commons Attribution 4.0 International License, which permits use, sharing, adaptation, distribution and reproduction in any medium or format, as long as you give appropriate credit to the original author(s) and the source, provide a link to the Creative Commons licence, and indicate if changes were made. The images or other third party material in this article are included in the article's Creative Commons licence, unless indicated otherwise in a credit line to the material. If material is not included in the article's Creative Commons licence and your intended use is not permitted by statutory regulation or exceeds the permitted use, you will need to obtain permission directly from the copyright holder. To view a copy of this licence, visit http://creativecommons.org/licenses/by/4.0/. The Creative Commons Public Domain Dedication waiver (http://creativeco mmons.org/publicdomain/zero/1.0/) applies to the data made available in this article, unless otherwise stated in a credit line to the data. 
and fulvestrant combination therapy as control data for further development of anti-HER2 agents plus hormone therapy.

Keywords: Trastuzumab, Fulvestrant, Hormone receptor-positive HER2-positive breast cancer

\section{Background}

Breast cancer is the most common cancer among women and the second most frequent newly diagnosed cancer worldwide [1]. Breast cancer has been divided into subtypes depending on the presence of hormone receptors (HRs) for estrogen and progesterone and HER2 expression [2]. Accordingly, there are four major subtypes of breast cancer: HR-positive HER2-negative (HR + HER2-), HR-positive HER2positive (HR + HER2+), HR-negative HER2-positive (HR-HER2+), and HR-negative HER2-negative (triple-negative breast cancer). The HER2-positive subtype accounts for $15-20 \%$ of all breast cancer subtypes [3]. Approximately half of the HER2-positive breast cancers express HRs [4]. HR + HER2+ breast cancer account for approximately $10 \%$ of all breast cancer cases $[5,6]$.

The standard first-line systemic treatment for metastatic HER2-positive breast cancer is chemotherapy with pertuzumab plus trastuzumab plus taxane [7]. Endocrine plus HER2-targeted therapy is a treatment option for patients with HR + HER2+ metastatic breast cancer. Previous clinical trials have showed that a combination of aromatase inhibitor and HER2-targeted therapy has greater clinical benefits than endocrine therapy alone in patients with HR+HER2+ breast cancer [8-10]. In the case of disease progression under aromatase inhibitor and HER2-targeted combination therapy, fulvestrant with HER2-targeted therapy is a reasonable treatment option; however, to our knowledge, the efficacy of fulvestrant and trastuzumab combination therapy has not been reported. Here, we retrospectively reviewed the efficacy and safety of fulvestrant and trastuzumab combination therapy at our hospital.

\section{Methods}

This study was designed as a retrospective review of the medical records of patients with advanced breast cancer who received systemic therapy between January 2001 and September 2020 at our institution. Patients with HR + HER2+ metastatic breast cancer were selected and those treated with fulvestrant and trastuzumab combination therapy were identified. HR and HER2 were assessed using tumor immunohistochemistry (IHC) and/or fluorescence in situ hybridization. Clinicopathological data, including age, HR and HER2 status, clinical and pathological stages, prior treatment of recurrent or metastatic breast cancer, metastatic organ site, response, treatment outcome, and survival, were collected from the medical records. Tumors were defined as HR + when IHC showed an Allred score $\geq 3$ for estrogen and/or progesterone receptor, according to American Society of Clinical Oncology/College of American Pathologists Guideline [11]. Tumor HER2 status was defined as positive when IHC for HER2 showed a score of $3+$ or $2+$ with gene amplification confirmation by fluorescence in situ hybridization. Patients received trastuzumab intravenously at $8 \mathrm{mg} / \mathrm{kg}$ on day 1 of cycle 1 , and this was maintained at $6 \mathrm{mg} / \mathrm{kg}$ on day 1 of all subsequent 21-day cycles. Fulvestrant was intramuscularly administered at $500 \mathrm{mg}$ on days 1 and 15 of cycle 1 and then once every 4 weeks.

\section{Endpoints}

The primary endpoint of this study was progression-free survival (PFS), and the secondary endpoints were overall survival (OS) and safety. In the analysis of survival data, 95\% CIs were estimated using the exact method. To evaluate the association between survival data and clinicopathological features, the chi-square test was used. A $P$ value of $<0.05$ was considered statistically significant. Statistical analysis was performed using JMP version 14.3.0 (SAS Institute Inc., Cary, NC, USA) and SPSS Statistics 27.0 (IBM corporation, New York, USA). This study was approved by the institutional review board (\# 2020-1280). Comprehensive consent was obtained from accusable patients in our institution.

\section{Results}

The data of 1612 patients with recurrent or metastatic breast cancer during the targeted period were reviewed and 118 patients with histologically documented HR + HER2+ breast cancer were identified. Of these, 28 patients received trastuzumab and fulvestrant combination therapy.

The patient characteristics are presented in Table 1. The median age was 66 (range, 50-80) years. Of these 28 patients, 7 (25\%) had de novo stage IV and 21 (75\%) had recurrent disease. Before trastuzumab and fulvestrant combination therapy, the median treatment line for advanced breast cancer was 6 . Six patients had a treatment line of $\leq 2$, and the remaining 22 patients were treated on the third treatment line or later. Trastuzumab was previously administered for advanced breast cancer to all patients, pertuzumab to four patients, and trastuzumab emtansine (T-DM1) to 10 patients. Of all patients, 
Table 1 Patient characteristics

\begin{tabular}{|c|c|}
\hline Characteristics & $N=\mathbf{2 8}(\%)$ \\
\hline \multicolumn{2}{|l|}{ Age (years) } \\
\hline median & 66 (range: $50-80$ ) \\
\hline \multicolumn{2}{|l|}{ Stage } \\
\hline De novo stage IV & $7(25)$ \\
\hline recurrent disease & $21(75)$ \\
\hline \multicolumn{2}{|l|}{ Subtype } \\
\hline $\mathrm{HR}+\mathrm{HER} 2+$ & $28(100)$ \\
\hline others & 0 \\
\hline \multicolumn{2}{|l|}{ Number of treatment line } \\
\hline median & 6 \\
\hline 1 or 2 & $6(21)$ \\
\hline $3-6$ & $9(32)$ \\
\hline $7-10$ & $9(32)$ \\
\hline$>10$ & $4(14)$ \\
\hline \multicolumn{2}{|c|}{ Prior treatment for recurrent or metastatic breast cancer } \\
\hline Trastuzumab & $28(100)$ \\
\hline Pertuzumab & $4(14)$ \\
\hline T-DM1 & $10(36)$ \\
\hline Trastuzumab deruxtecan (T-DXd) & $2(7)$ \\
\hline \multicolumn{2}{|l|}{ Visceral metastasis } \\
\hline Yes & $21(75)$ \\
\hline No & $7(25)$ \\
\hline \multicolumn{2}{|l|}{ Liver metastasis } \\
\hline Yes & $11(39)$ \\
\hline No & $17(61)$ \\
\hline \multicolumn{2}{|l|}{ Brain metastasis } \\
\hline Yes & $4(14)$ \\
\hline No & $24(86)$ \\
\hline
\end{tabular}

21 (75\%) had visceral metastasis, including liver and brain metastases.

\section{Efficacy}

Complete response (CR) was not observed in any patient. Partial response (PR) was observed in 1, stable disease (SD) in 17 , and progressive disease in 10 patients. The overall response rate was $4 \%$, and the disease control rate $(\mathrm{DCR}=\mathrm{CR}+\mathrm{PR}+\mathrm{SD})$ was $64 \%$. The median PFS was 6.4 months (95\% CI, 3.46-8.17), and median OS was 35.3 months (95\% CI, 20.0-46.7) (Figs. 1 and 2). Subgroup analysis depending on patient characteristics is shown in Fig. 3, which suggested that no significant difference in PFS was observed in any subgroup analysis. PFS in patients who received trastuzumab and fulvestrant combination therapy as $\leq 3 \mathrm{rd}$ line treatment or did not have liver metastasis tended to be better compared to those in patients who received the treatment in later line or with liver metastasis. Median PFS was 7.3 months (95\% CI, 1.53-18.67) and median OS was not achieved in patients receiving trastuzumab and fulvestrant combination therapy as $\leq 3$ rd line treatment. In contrast, the median PFS was 5.2 months (95\% CI, 2.57-8.17), and median OS was 34.8 months (95\% CI, 14.5-45.1) in patients who received the therapy as $>3$ rd line treatment. The PFS and OS of this subgroup were not significantly different (log-rank test, $p=0.4299$ and $p=0.2550$, respectively) (PFS data in Fig. 4). Additionally, PFS in patients with liver metastasis tended to be worse than that in patients without liver metastasis, and median PFS was 3.5 months (95\% CI, 1.17-8.93) and 6.9 months (95\% CI, 3.93-8.17), respectively (log rank test, $p=0.3573$ ) (Fig. 5). No adverse events of $\geq$ grade 3 were observed.

\section{Discussion}

Trastuzumab and fulvestrant combination therapy showed a median PFS of 6.4 months, median OS of 35.3 months, and 64\% DCR, which suggested moderate efficacy in patients with HR + HER2+ metastatic breast cancer. In this study, most patients were heavily pretreated and developed visceral metastasis, including liver and brain metastases, indicating poor prognosis in these patients. The response rate was only $4 \%$, which might be due to the late treatment line of this population; the median number of treatment lines was six, and four patients were treated after the 10th line.

Real-world evidence of treatment after T-DM1 in patients with HER2+ metastatic breast cancer showed that most patients received trastuzumab and chemotherapy after T-DM1, and approximately $5 \%$ of the patients received hormone therapy with or without anti-HER2 antibodies [12]. The median PFS after T-DM1 treatment was 6.1 months (95\% CI, 5.3-6.7) and median OS was 23.7 (95\% CI, 20.7-27.4), which are comparable with the PFS and OS of trastuzumab and fulvestrant combination therapy in the current study. This suggests that trastuzumab and fulvestrant combination therapy is a reasonable treatment option after T-DM1 for patients with $\mathrm{HR}+\mathrm{HER} 2+$ metastatic breast cancer.

Recently, clinical trials on the addition of a CDK4/6 inhibitor to the combination of fulvestrant and HER2targeted therapy have been conducted in patients with HR + HER2 + breast cancer $[13,14]$. However, the data on fulvestrant and trastuzumab combination therapy that should have been the background for these studies are scarce; hence, our data are considered useful. The monarcHER trial, a phase 2 trial that compared abemaciclib plus trastuzumab plus fulvestrant, abemaciclib plus trastuzumab, and standard-of-care chemotherapy plus trastuzumab, showed better PFS with abemaciclib plus trastuzumab plus fulvestrant than with standard-of-care chemotherapy plus trastuzumab [13]. The median PFS of the abemaciclib plus trastuzumab plus fulvestrant group 


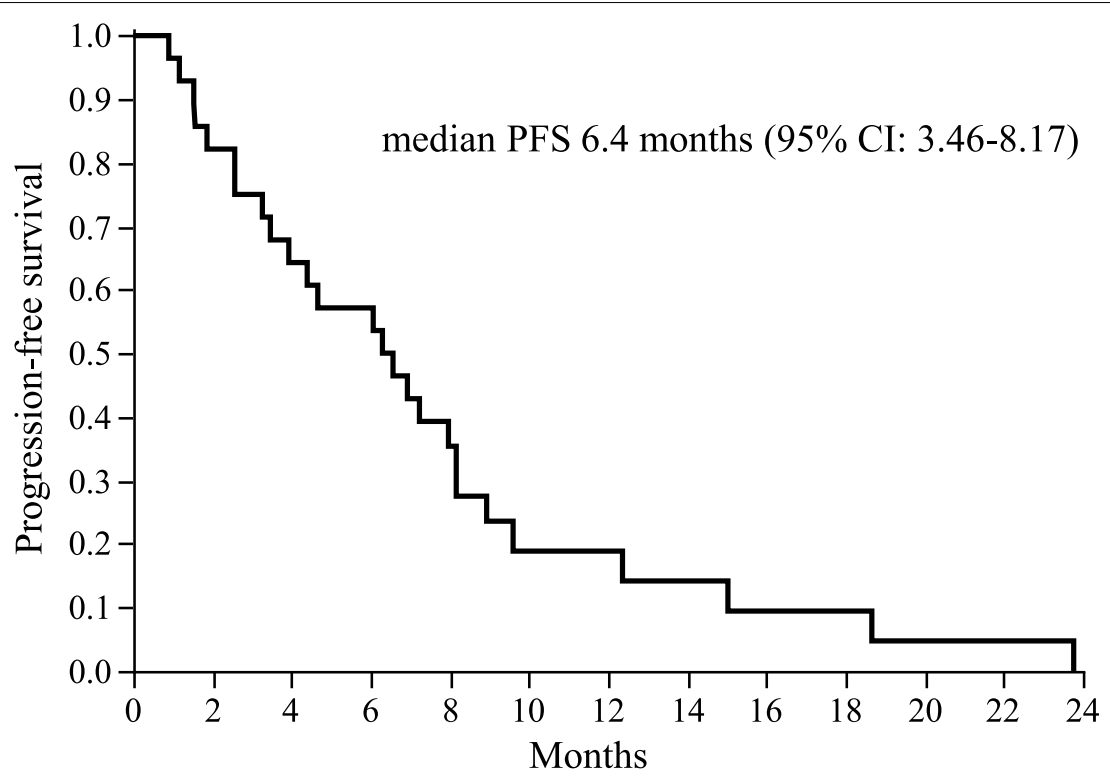

$\begin{array}{lllllllllllll}\text { No. at risk } & 28 & 23 & 18 & 16 & 9 & 4 & 4 & 3 & 2 & 2 & 1 & 1\end{array}$

Fig. 1 Progression-free survival in all patients. Kaplan-Meier curve of progression-free survival (PFS) of fulvestrant and trastuzumab combination therapy in patients with hormone receptor- and human epidermal growth factor receptor 2-positive metastatic breast cancer

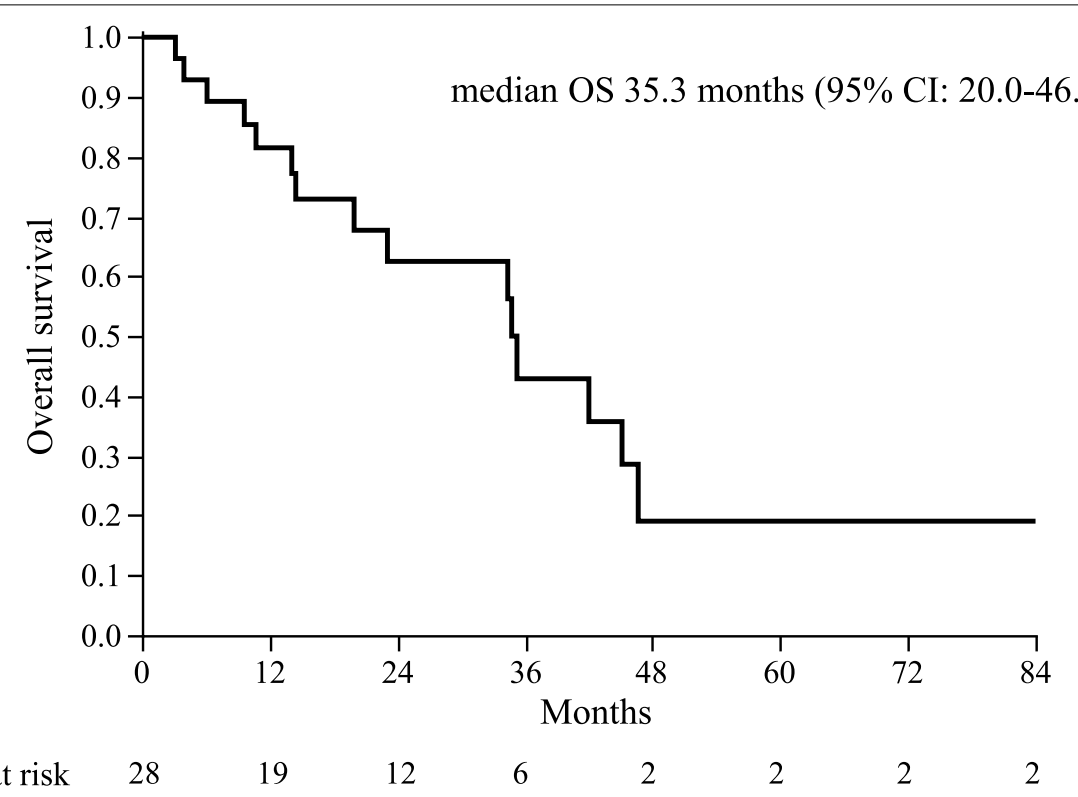

Fig. 2 Overall survival in all patients. Kaplan-Meier curve of OS of fulvestrant and trastuzumab combination therapy in patients with hormone receptor- and human epidermal growth factor receptor 2-positive metastatic breast cancer

$(n=79)$ and that of standard-of-care chemotherapy and trastuzumab group were 8.3 months (95\% CI, 5.9-12.6) and 5.7 (95\% CI, 5.4-7.0), respectively. The PFS of trastuzumab plus fulvestrant in our study was comparable to that of standard-of-care chemotherapy and trastuzumab in the monarcHER trial. The patient background in our study was also similar to that in the monarcHER trial. For example, patients who received at least two HER2targeted therapies for advanced breast cancer were included. In the abemaciclib plus trastuzumab plus fulvestrant group, $44 \%$ patients had two or three previous systemic therapies for advanced breast cancer and 56\% 

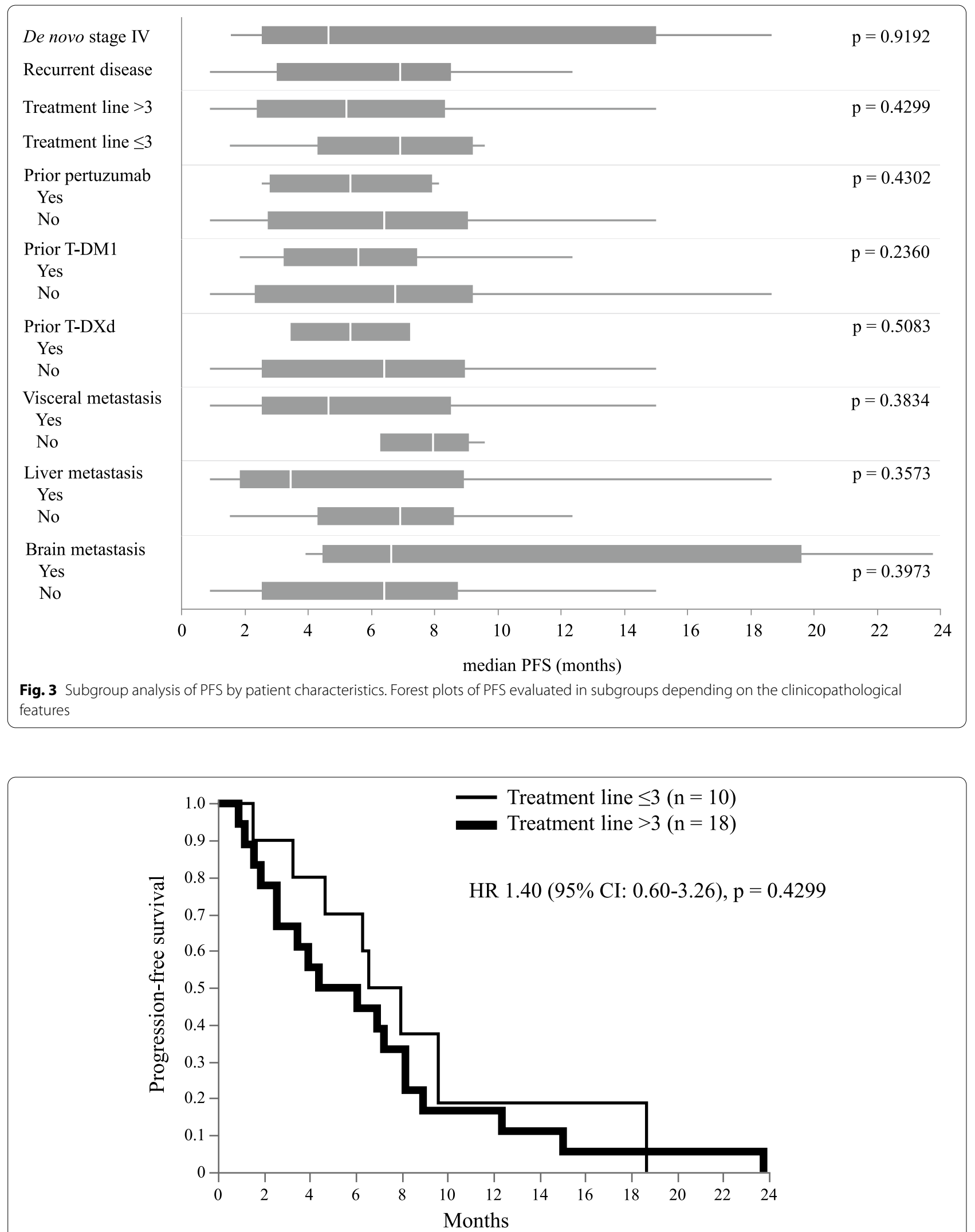

Fig. 4 PFS subgroup analysis by number of treatment line. Kaplan-Meier curves of PFS in patients who received fulvestrant and trastuzumab combination therapy as $\leq 3$ line treatment or $>3$ line treatment 


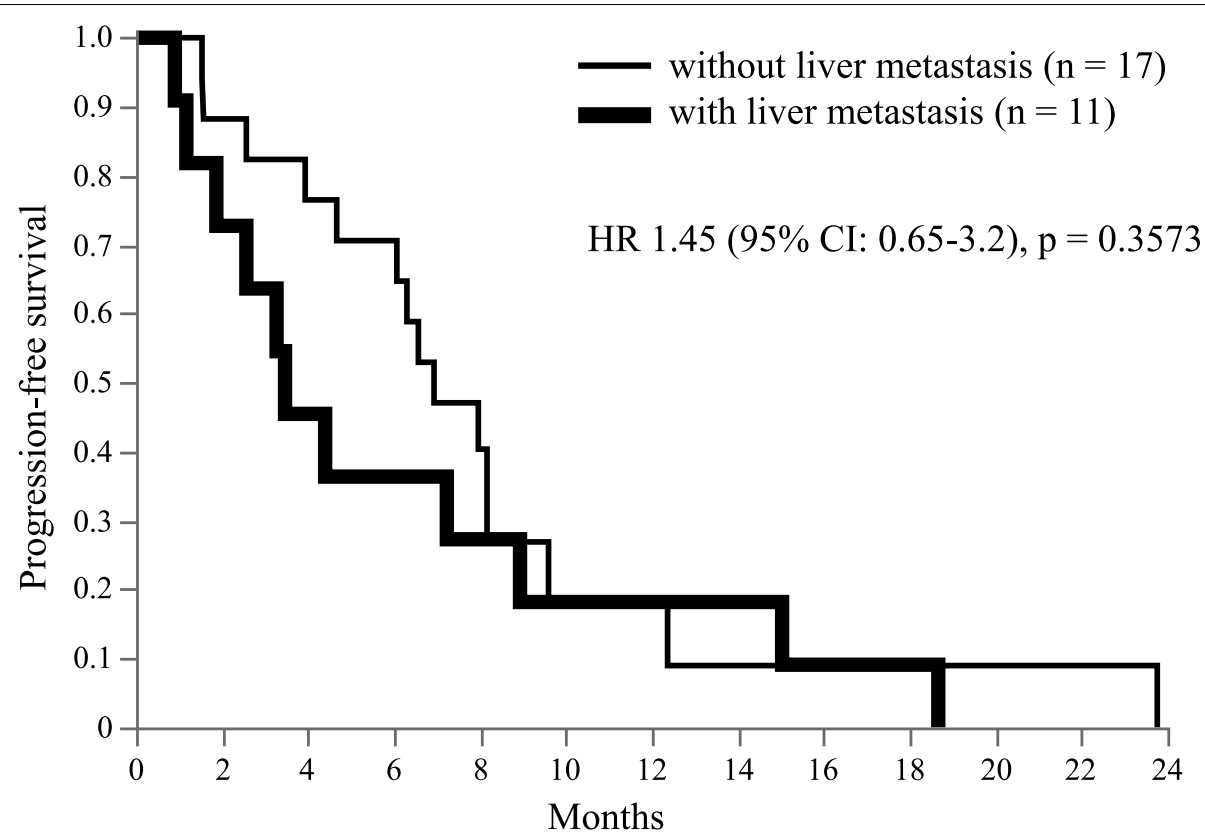

Fig. 5 PFS subgroup analysis in patients with or without liver metastasis. Kaplan-Meier curves of PFS in patients who had liver metastasis or not

had four or more. Moreover, $73 \%$ patients had visceral metastasis, and most patients were heavily pretreated for it. The overall response rate in the abemaciclib plus trastuzumab plus fulvestrant group was $33 \%$, which was higher than that in our survey. This suggests that adding abemaciclib to trastuzumab and fulvestrant combination therapy in this population is effective. Based on these efficacy data, anti-HER2 therapy plus endocrine plus CDK4/6 inhibitor combination is a promising strategy in this patient population and phase 3 trials are ongoing. For example, DETECT V/CHEVENDO trial is recruiting patients, which is a randomized phase III study comparing the safety and efficacy of trastuzumab plus pertuzumab and the ribociclib, CDK4/6 inhibitor, along with either endocrine therapy or chemotherapy and includes trastuzumab plus pertuzumab plus ribociclib plus fulvestrant arm (NCT02344472). Another phase III trial, PATINA, is evaluating the efficacy of adding palbociclib to the anti-HER2 therapy plus endocrine therapy after 4 to 8 cycles of induction treatment (NCT02947685).

There has recently been remarkable progress in the development of new drugs for HER2+ metastatic breast cancer [15]. These new drugs include novel antibodydrug conjugates (trastuzumab deruxtecan) and tyrosine kinase inhibitors (neratinib and tucatinib). Some of these agents are being developed in clinical trials as combination therapy with fulvestrant; however, there are no control efficacy data on trastuzumab and fulvestrant combination therapy. This study contributes to understanding the efficacy of trastuzumab and fulvestrant combination therapy and further development of antiHER2 agents plus hormone therapy.

We recognized several imitations of this study. First, this is a single-center retrospective study, which makes it difficult to appropriately evaluate adverse events or toxicity. Second, only $14 \%$ patients previously received treatment containing pertuzumab. The records include patients since 2001, which implies that most patients received anti-HER2 treatment before pertuzumab approval. Finally, the number of patients who received trastuzumab and fulvestrant was small, which requires careful interpretation of the results.

\section{Conclusions}

Trastuzumab and fulvestrant combination therapy showed moderate clinical efficacy, which is a reasonable option for HR + HER2+ metastatic breast cancer after standard anti-HER2 therapy. These data contribute to understanding the efficacy of trastuzumab and fulvestrant combination therapy as control data for further development of anti-HER2 agents plus hormone therapy.

\section{Abbreviations}

HER2: Human epidermal growth factor receptor 2; Cl: Confidence interval; HR: Hormone receptor; IHC: Immunohistochemistry; PFS: Progression-free survival; OS: Overall survival; T-DM1: Trastuzumab emtansine; CR: Complete response; PR: Partial response; SD: Stable disease.

\section{Acknowledgements}

We thank the participants and their families who contributed to this study. We thank Edanz Group (https://en-author-services.edanz.com/ac) for editing a draft of this manuscript. 


\section{Authors' contributions}

YO, YA, JM, LI, SK, TS, TM, and MK analyzed and interpreted the patient data. $\mathrm{KY}, \mathrm{SS}, \mathrm{MH}$, IF and FH performed the data collection. TK, KK, SM, TT, TU and SO were major contributors in writing and reviewing the manuscript. All authors read and approved the final manuscript.

\section{Funding}

No funding for this study.

\section{Availability of data and materials}

The datasets used and/or analyzed during the current study available from the corresponding author on reasonable request.

\section{Declarations}

\section{Ethics approval and consent to participate}

Comprehensive consent was obtained from accusable patients in our institution.

Besides comprehensive consent, specific informed consent for this study was not required because this was a retrospective study with an opt-out option. The protocol of this study was approved and informed consent was waived by the institutional review board (committee member: Kohei Omatsu. Gynecologic Oncology Department) of the Cancer Institute Hospital of Japanese Foundation for Cancer Research, Tokyo, Japan (approved number: \# 2020-1280).

This study had conducted in accordance with ethical guidelines for medical and health research involving human subjects.

\section{Consent for publication}

No personal information is included.

Not applicable.

\section{Competing interests}

The authors have competing interests in relation to this study. Competing interests are listed below.

Y. Ozaki reports personal fees from Chugai.

T. Takano reports grants and personal fees from Daiichi-Sankyo, Kyowa Kirin, Eisai, and Chugai; grants from Ono Pharmaceutical, Bristol-Myers Squibb, MSD, Merck Serono, Taiho, and Novartis; and personal fees from Pfizer, Eli Lilly, and Celltrion.

F. Hara reports grants, personal fees from Chugai, Pfzer, Kyowa Kirin, Eli Lilly Japan.

T. Ueno received personal fees from Chugai, AstraZeneca, Taiho, and Novartis and grants and personal fees from Eisai.

No COl of other co-authors.

\section{Author details}

${ }^{1}$ Breast Oncology Center, The Cancer Institute Hospital of Japanese Foundation for Cancer Research, 3-8-31 Ariake, Koto-ku, Tokyo 135-8550, Japan.

${ }^{2}$ Department of Clinical Oncology, Graduate School of Medical and Dental Sciences, Tokyo Medical and Dental University, Tokyo, Japan. ${ }^{3}$ Department of Medical Oncology, Toranomon Hospital, Tokyo, Japan.

Received: 15 February 2021 Accepted: 16 December 2021

Published online: 04 January 2022

\section{References}

1. Kolak A, Kaminska M, Sygit K, Budny A, Surdyka D, Kukielka-Budny B, et al. Primary and secondary prevention of breast cancer. Ann Agric Environ Med. 2017;24(4):549-53.

2. lancu G, Vasile D, lancu RC. DaviToiu DV: "triple positive" breast cancer - a novel category? Romanian J Morphol Embryol. 2017;58(1):21-6.

3. Nayar U, Cohen O, Kapstad C, Cuoco MS, Waks AG, Wander SA, et al. Acquired HER2 mutations in ER(+) metastatic breast cancer confer resistance to estrogen receptor-directed therapies. Nat Genet. 2019;51(2):207-16.
4. Schedin TB, Borges VF, Shagisultanova E. Overcoming therapeutic resistance of triple positive breast Cancer with CDK4/6 inhibition. Int J Breast Cancer. 2018:2018:7835095.

5. Kast K, Link T, Friedrich K, Petzold A, Niedostatek A, Schoffer O, et al. Impact of breast cancer subtypes and patterns of metastasis on outcome. Breast Cancer Res Treat. 2015;150(3):621-9.

6. Howlader N, Altekruse SF, Li Cl, Chen VW, Clarke CA, Ries LA, et al. US incidence of breast cancer subtypes defined by joint hormone receptor and HER2 status. J Natl Cancer Inst. 2014;106:5

7. Swain SM, Miles D, Kim SB, Im YH, Im SA, Semiglazov V, et al. Pertuzumab, trastuzumab, and docetaxel for HER2-positive metastatic breast cancer (CLEOPATRA): end-of-study results from a double-blind, randomised, placebo-controlled, phase 3 study. Lancet Oncol. 2020;21(4):519-30.

8. Kaufman B, Mackey JR, Clemens MR, Bapsy PP, Vaid A, Wardley A, et al. Trastuzumab plus anastrozole versus anastrozole alone for the treatment of postmenopausal women with human epidermal growth factor receptor 2-positive, hormone receptor-positive metastatic breast cancer: results from the randomized phase III TAnDEM study. J Clin Oncol. 2009;27(33):5529-37.

9. Huober J, Fasching PA, Barsoum M, Petruzelka L, Wallwiener D, Thomssen $C$, et al. Higher efficacy of letrozole in combination with trastuzumab compared to letrozole monotherapy as first-line treatment in patients with HER2-positive, hormone-receptor-positive metastatic breast cancer - results of the eLECTRA trial. Breast. 2012;21(1):27-33.

10. Johnston S, Pippen J Jr, Pivot X, Lichinitser M, Sadeghi S, Dieras V, et al. Lapatinib combined with letrozole versus letrozole and placebo as firstline therapy for postmenopausal hormone receptor-positive metastatic breast cancer. J Clin Oncol. 2009;27(33):5538-46.

11. Hammond ME, Hayes DF, Dowsett M, Allred DC, Hagerty KL, Badve S, et al American Society of Clinical Oncology/College of American Pathologists guideline recommendations for immunohistochemical testing of estrogen and progesterone receptors in breast cancer (unabridged version). Arch Pathol Lab Med. 2010;134(7):e48-72.

12. Yokoe T, Kurozumi S, Nozawa K, Ozaki Y, Maeda T, Yazaki S, et al. Clinical benefit of treatment after trastuzumab emtansine for HER2-positive metastatic breast cancer: a real-world multi-Centre cohort study in Japan (WJOG12519B). Breast Cancer. 2021.

13. Tolaney SM, Wardley AM, Zambelli S, Hilton JF, Troso-Sandoval TA, Ricci F, et al. Abemaciclib plus trastuzumab with or without fulvestrant versus trastuzumab plus standard-of-care chemotherapy in women with hormone receptor-positive, HER2-positive advanced breast cancer (monarcHER): a randomised, open-label, phase 2 trial. Lancet Oncol. 2020;21(6):763-75.

14. Gianni L, Bisagni G, Colleoni M, Del Mastro L, Zamagni C, Mansutti M, et al. Neoadjuvant treatment with trastuzumab and pertuzumab plus palbociclib and fulvestrant in HER2-positive, ER-positive breast cancer (NA-PHER2): an exploratory, open-label, phase 2 study. Lancet Oncol. 2018;19(2):249-56.

15. Ponde N, Brandao M, El-Hachem G, Werbrouck E, Piccart M. Treatment of advanced HER2-positive breast cancer: 2018 and beyond. Cancer Treat Rev. 2018;67:10-20.

\section{Publisher's Note}

Springer Nature remains neutral with regard to jurisdictional claims in published maps and institutional affiliations. 